of three batch chargers over a period of $\frac{1}{2} \mathrm{hr}$. From 35 to 350 mc. of phosphorus-32 was used in an investigation, $175 \mathrm{mc}$. being found to be the optimum activity.

Samples were taken at intervals from the output of three bottle-making machines fed by the furnace and measured with an end-window Geiger counter. By using a metal iris to limit the area inspected by the counter and by using glass samples thicker than $3 \mathrm{~mm}$., it was established that the shape of the samples did not critically affect the results, and pieces of bottles were conveniently used for messurements.

It was found that tracer added to one of the outside batch chargers appeared almost uniformly in the three bottle-making machines, indicating good mixing and absence of streaming in the furnace. After a delay of $7 \frac{1}{2}-10 \mathrm{hr}$. for amber glass and 5-6 hr. for flint, radioactivity began to appear in the product, reaching a maximum in about $24 \mathrm{hr}$. This was followed by a nearly exponential decay, leaving only 1.4 per cent of the labelled glass after 12 days and undetectable activity after 14 days. Apart from the expected initial delay before marked constituents appeared in the product, agreement with the 'curve of perfect mixing' was fairly good. Some subsidiary peaks in the curve of activity versus time were unexplained, but some were believed to be due to the recycling of active cullet.

Mr. R. F. Barker (Pilkington Brothers, Ltd.) gave an account of an "Investigation of Glass Flow in Furnaces by Radioactivation Methods". The furnace described was producing $6 \cdot 4$ tons per $h r$. of soda lime silica glass and had a capacity of 1,350 tons. Messurements were required over a period of several weeks. The long half-life and high activity required in a radioactive tracer made the use of radioactivity in the furnace undesirable. Hence an inactive material which could be made radioactive by the subsequent neutron irradiation of samples was used. Tantalum was chosen because of its high neutron cross-section and the desirability of the gamma-emitting tantalum182 formed on neutron irradiation, the half-life (110 days) of which was longer than that of any activity induced in the normal constituents of the glass. The tracer was in the form of $3 \frac{8}{6} \mathrm{kgm}$. of tantalum peroxide.

Tests were made on three of five machines fed by the furnace, and 200 samples, each containing $5 \mathrm{gm}$. of powdered glass, were irradiated in a reactor at Harwell. After a delay of five weeks to allow shortlived activities to decay, measurements were made with a scintillation counter, using a single-channel pulse amplitude analyser to distinguish the tantelum. 182 gamma rays. A minimum transit-time of $4 \mathrm{hr}$. was found, in agreement with other data. The tracer was at a maximum after $100 \mathrm{hr}$. and its decline was followed over a total time of $\mathbf{5 2 0}$ furnace-hours. Moderate agreement was found with W. M. Hampton's theoretical curve for perfect mixing, but Mr. Barker directed attention to significant departures from this curve near the time of injection.

In a second paper, on "The Assessment by Tracer Methods of Mixing during Sand Treatment", Mr. R. F. Barker deseribed experiments made in collaboration with the Isotope Division, Harwell, using sand labelled by immersion in a solution of barium140 and subsequent heating at $500^{\circ} \mathrm{C}$. The adhesion of the radioactive material was already established under conditions of abrasion and washing with water. The daughter isotope lanthanum-140 (40 hr.) reaches equilibrium with the barium-140 (12 days) and measurements were made of gamma rays from the lanthanum.

Ten pounds of labelled sand, carrying $100 \mathrm{me}$. of barium-140, were mixed with 5 cwt. of inactive sand in a cement mixer and added to a hopper feeding the washing plant. The progress of the radioactive material through the washing plant and grader was registered by recording meters placed outside the steel pipes carrying the sand slurry. A survey was made of the horizontal distribution of radioactively labelled sand in the storage bin (capacity 2,000 tons). At this stage the labelled and was found still to be fairly localized. Further monitoring of the sand leaving the bin and in the railway transit wagons showed that mixing was introduced by the method of loading.

Finally, timed measurements were made of the radioactivity of glass drawn off from the tank furnace. From an analysis of these results the distribution of a particular sample of the original sand in the finished product was assessed.

The papers were followed by lively discussion. The proceedings of the symposium will be published in the Journal of the Society of Glass Technology.

J. L. PUTMan

\title{
MELTING AND DIFFUSION
}

\begin{abstract}
$\mathrm{T}$ HE socond symposium on "Melting, Diffusion and Related Topics" was held at the National Research Council Laboratories, Ottawa, on October 21-22. The meeting was sponsored by the Low Temperature and Solid State Physics Group of the Division of Pure Physics, National Research Council of Canada. As in the previous symposium, reported in Nature $(177,23 ; 1956)$, the object was to bring together workers with varied approaches to the subject and, by limiting the number of formal contributions to twelve, to encourage informal discussion.

In the previous symposium the melting process itself was a major topic of interest. In contrast, the programme on this occasion was concerned chiefly with processes having only a general bearing on melting, the direct approach being temporarily
\end{abstract}

abandoned in favour of seeking clues from the solution of associated problems.

Among the papers concerned with measurements in the region of melting was one by D. L. Martin (National Research Council), who reviewed existing information concerning specific heat anomalies in the region of melting ; he showed that both anharmonicity and lattice defects have been invoked in explanation but argued that little conclusive evidence exists as to their relative importance. N. H. Nachtrieb (Institute of Metals, Chicago) dealt with diffusion in liquid and solid pure metals and in alloys; the analysis of his measurements showed a clear relation between melting and diffusion parameters, but also underlined the restricted nature of purely thermodynamic arguments. Diffusion was also considered 
by J. A. Morrison and R. Rudham (National Research Council), who gave details of their identification of the mechanism of the anion diffusion process in sodium and potassium chloride crystals. Slow neutron diffraction measurements were described by a group of workers from Chalk River : D. G. Henshaw and N. K. Pope gave complementary experimental and theoretical accounts of the determination of the radial distribution function in solid and liquid helium and neon and in liquid argon, while B. N. Brockhouse showed how the incoherent scattering could give information on molecular motions and may provide a means of resolving some of the indeterminancies involved in the direct measurement of diffusion.

A second group of contributions dealt with transitions other than melting. R. W. Smith (University of Toronto) described investigations on the grey tin/white tin transformation carried out in Birmingham with G. V. Raynor, in which previous uncertainties of observation have been positively correlated with impurity content. D. Gugan and J. S. Dugdale (National Research Council) gave a preliminary report of work in which electrical resistivity measurements appear to provide informa. tion on the martensitic transformation in lithium.
Z. S. Basinski (National Research Council) outlined a theory of work hardening of face-centred cubic metals in which the main obstacles were provided by the 'forest' of dislocations; the theory appeared to apply also to body-centred cubic metals, but in that case at low temperatures the mechanical properties were dominated by a large Peierls-Nabarro force. Work on the liquid state was represented by an account given by A. C. Hollis Hallett (University of Toronto) of recent experiments on the viscosity of liquid helium.

The single contribution dealing specifically with melting was by J. S. Dugdale, who discussed the Lindemann melting formula in relation to inert gas solids. R. J. Uffen (University of Western Ontario) once again made a convincing defence of large-scale approximation and extrapolation, this time discussing certain tectonic and volcanic phenomena in terms of the pressure dependence of melting of various types of magma.

In these days of high-speed conferences it is pleasurable to report on one at which the pace and length of discussion were such as to enable at least one non-expert to participate with profit. An informal report of the proceedings is in preparation. J. W. LEECH

\section{ELECTRONICALLY EXCITED SPECIES}

A SYMPOSIUM on "The Structure and Reactivity of Electronically Excited Species" was held at Ottawa during September 5-6 under the auspices of the Physical Chemistry Division of the Chemical Institute of Canada.

In his introduction to the section on structure, G. Herzberg directed attention to the rapid increase, recently, in the number of polyatomic molecules, radicals and ions for which rotational and vibrational analyses of electronic systems had been carried out. This had demonstrated that, in many cases, the shapes in the excited states are different from those of the groundstates. For example, acetylene, hydrogen cyanide and carbon disulphide are linear in their groundstates but bent in their first electronically excited states. Throughout the meeting it was clear that the predictions made by A. D. Walsh in 1953 had been substantiated most impressively. References were repeatedly made to Walsh's energy diagrams.

G. W. Robinson and V. E. DiGiorgio showed in a submitted paper that formaldehyde is non-planar in a low-lying excited state, while $\mathbf{K}$. Allison and A. D. Walsh concluded that the same may be true for one or two of the excited states involved in the Rydberg transitions. G. W. King presented evidence for the conclusion that there is only a small change in molecular geometry on exciting the glyoxal molecule in the transition studied by him.

During this part of the meeting much interest was shown in the interactions between electronic and vibrational motions of polyatomic molecules. M. H. L. Pryce, U. Opik, H. C. Longuet-Higgins and R. A. Sack dealt theoretically with the dynamical JahnTeller effect, considering the energy-levels of a nonlinear molecule in a doubly degenerate electronic state which is split by a doubly degenerate vibrational mode. K. Dressler and D. A. Ramsay dealt with the Renner effect in linear molecules. The lowest state of linear $\mathrm{NH}_{2}$ would be a II state. Experimental results suggest that the ground-state is bent and the first excited state is linear, the vibrational levels of the hypothetical linear state correlating with the levels of those two states, half with those of the excited and half with those of the ground-state. The ground- and excited states are degenerate when the radical is linear but different in energy when it is bent. The radical HCO appears to behave similarly.

Two papers by J. N. Murrell and by D. S. McClure were submitted which discussed the transfer of electronic and vibrational energy between weakly coupled systems. This is a subject of very great interest at the moment.

In addition to molecules and radicals the excited states of ions were discussed during the meeting. D. C. Frost and C. A. McDowell described their mass spectrometer which has been employed to determine the energies of the excited states of the ions produced. in the instrument. This complements information that can be obtained spectroscopically, though so far detailed analyses of polyatomic ionic spectra are only available for $\mathrm{CO}_{2}+, \mathrm{CS}_{2}+$ and $\mathrm{C}_{4} \mathrm{H}_{2}+$. Also J. W. Sidman, in an interesting paper, described results obtrined for the nitrite ion in the crystalline state. From these he obtained information concerning the vibrational and lattice frequencies of the groundand excited states.

There is doubt about the multiplicities of the excited states of some molecules and, in this connexion, A. E. Douglas presented some results for the Zeeman effect in the near ultra-violet bands of carbon disulphide. This indicates that the excited state is a triplet. G. W. Robinson and V. E. DiGiorgio concluded that the weak bands of formaldehyde studies by them were due to a singlettriplet system. 